\title{
Proniosomal powders of natural canthaxanthin: preparation and characterization
}

Running title: Proniosomal powders of natural canthaxanthin

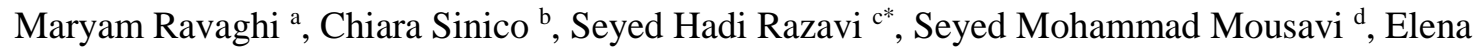
Pini $^{\mathrm{e}}$, Anna Maria Fadda ${ }^{\mathrm{f}}$

${ }^{a}$ Department of Food Science, Engineering and Technology, Faculty of Agricultural Engineering and Technology, University of Tehran, P.O. Box 4111, Karaj 3158777871, Iran.

ravaghi.maryam@ut.ac.ir

${ }^{\mathrm{b}}$ Department of Life and Environmental Sciences, University of Cagliari, Via Ospedale 72, 09124

Cagliari, Italy.sinico@unica.it

${ }^{\mathrm{c}}$ Center of Excellence for Application of Modern Technologies for Producing Functional Foods and Drinks and Bioprocess Engineering Laboratory (BPEL), Department of Food Science, Engineering and Technology, Faculty of Agricultural Engineering and Technology, University of Tehran, P.O. Box 4111, Karaj 3158777871, Iran. srazavi@ut.ac.ir

${ }^{\mathrm{d}}$ Center of Excellence for Application of Modern Technologies for Producing Functional Foods and Drinks and Bioprocess Engineering Laboratory (BPEL), Department of Food Science, Engineering and Technology, Faculty of Agricultural Engineering and Technology, University of Tehran, P.O. Box 4111, Karaj 3158777871, Iran. mousavi@ut.ac.ir

*Corresponding author. Tel.: +98 263224 8804; fax: +98 263224 9453; Email address: srazavi@ut.ac.ir (S.H. Razavi).

(C) 2016. This manuscript version is made available under the Elsevier user license http://www.elsevier.com/open-access/userlicense/1.0/ 
${ }^{\mathrm{e}}$ Department of Pharmaceutical Science, University of Milan, Via Venezian 21, Milan, Italy. elena.pini@unimi.it

${ }^{\mathrm{f}}$ Department of Life and Environmental Sciences, University of Cagliari, Via Ospedale 72, 09124 Cagliari, Italy.mfadda@unica.it

Abbreviations: BPEL, Bioprocess Engineering Laboratory; CI, Carr's compressibility index; CR, Canthaxanthin recovery; CTX, Canthaxanthin; DE, Dextrose equivalent; DLS, Dynamic light scattering; DSC, Differential scanning calorimetry; FTIR, Fourier transform infrared spectroscopy; HPLC, High performance liquid chromatography; HR, Hausner's ratio; LA, Lactose; LAPP, Lactose based proniosome powder; MA, Mannitol; MAPP, Mannitol based proniosome powder; MD, Maltodextrin; MDPP, Maltodextrin based proniosome powder; MW, Mlecular weight; PDI, Polydispersity index; PU, Pullulan; PUPP, Pullulan based proniosome powder; PXRD, Powder X-ray diffraction; SEM, Scanning electron microscopy; TC, Total carotenoids; TEM, Transmission electron microscopy; YMA, Yeast malt extract agar. 


\begin{abstract}
In this study, canthaxanthin (CTX) was produced by Dietzia natronolimnaea HS-1 using beetroot molasses as substrate and used for encapsulation in proniosome powders after extraction, with the aim of improving its stability. Proniosome powders were prepared with an equimolar ratio of span 60/cholesterol and four different carriers, namely maltodextrin, mannitol, lactose and pullulan. The properties of these formulations as both proniosomal powders and resulted niosomal dispersions were evaluated. The type of carriers had significant effects on the micrometric properties of proniosome powders which were further confirmed by the results of SEM analysis. Although light and high temperatures affected the stability of CTX drastically, but encapsulation in proniosomes retarded its degradation. Among these samples, mannitol based proniosome powder (MAPP) produced small vesicles $($ mean diameter $=175 \pm 3 \mathrm{~nm}$ and polydispersity index $=0.28 \pm 0.02)$ with the highest entrapment efficiency $(74.1 \pm 2.7 \%)$. MAPP provided a promising formulation to increase CTX stability especially upon storage at high temperatures $\left(45^{\circ} \mathrm{C}\right)$.
\end{abstract}

Keywords: Canthaxanthin, Carrier, Dietzia natronolimnaea HS-1, Proniosome, Span 60.

Chemical compounds studied in this article

Canthaxanthin (PubChem CID: 5281227); Cholesterol (PubChem CID: 5997); Lactose (PubChem CID: 440995); Maltodextrin (PubChem CID: 107526); Mannitol (PubChem CID: 6251); Pullulan (PubChem CID: 92024139); Span 60 (PubChem CID: 16218600) 


\section{Introduction}

Carotenoids are yellow, orange or red pigments naturally present in all photosynthetic organisms and non photosynthetic bacteria and fungi (Gharibzahedi, Razavi, Mousavi, \& Moayedi, 2012; Tanaka, Shnimizu, \& Moriwaki, 2012). Vertebrates lack the ability to synthesize carotenoids and are dependent on dietary sources (Clark, Faustman, Chan, Furr, \& Riesen, 1999). In addition to their pigmenting abilities, carotenoids exhibit high pharmaceutical values and potential health benefits such as strengthening the immune system, decreasing the risk of degenerative illnesses, preventing the risk of cardiovascular disease and macular degeneration, reducing the risk of cataracts by quenching triplet sensitizers and singlet oxygen, and trapping free radicals (Clark et al., 1999; Gharibzahedi et al., 2012; Nasri Nasrabadi \& Razavi, 2010; Santos \& Meireles, 2010; Socaciu, 2007). Canthaxanthin (CTX) is a xanthophyll (a subclass of carotenoids) with widespread applications in the pharmaceutical, cosmetic, fishery, poultry and food industries (Gharibzahedi, Razavi, \& Mousavi, 2013c; Hojjati, Razavi, Rezaei, \& Gilani, 2014; Nasri Nasrabadi \& Razavi, 2010). It is a superior antioxidant and scavenger of free radicals as compared with carotenoids such as $\beta$-carotene (Tanaka et al., 2012). Although CTX, such as other polyene compounds, is known as lipid soluble, it can be partially water soluble due to little polarity of its terminal groups (Supplementary Figure 1) (Seyedrazi, Razavi, \& Emam-Djomeh, 2011). As most food matrices are water soluble, many efforts were directed to the formulation of lipophilic pigments (mainly carotenoids) into water soluble formulations (Socaciu, 2007).

Recently, production of this pigment from biological resources, with regard to side effects of synthetic carotenoids for human consumption and consumer preferences for functional 
food products, has been attended throughout the world (Ekin Gumus, Davidov-pardo, \& Julian McClements, 2016; Gharibzahedi, Razavi, \& Mousavi, 2013d; Hojjati, Razavi, Rezaei, \& Gilani, 2011). For the industrial production of carotenoids, microorganisms are preferred over other natural sources, such as vegetables and fruits, owing to problems of seasonal and geographic variability in production. In addition, there are economic advantages to microbial processes that use agricultural waste and industrial wastewater as substrates (Hojjati et al., 2014). Dietzia natronolimnaea HS-1 is one of the best microbial sources for CTX production in compared to the levels of CTX biosynthesized by other main wild CTX producing strains such as Brevibacterium KY-4313, Bradyrhizobium strain ORS278, Haloferax alexandrinus TMT, Micrococcus roseus, Gordonia jacobaea MV-1 and Chlorella zofingiensis (Gharibzahedi, Razavi, \& Mousavi, 2013a; Gharibzahedi et al., 2012). It is a Gram positive, catalase positive, and oxidase negative bacterium which can produce more than 90\% CTX of the total carotenoids (Gharibzahedi et al., 2013a).

Processing and preservation of products containing these pigments are extremely difficult due to their high sensitivity to operational and environmental conditions such as $\mathrm{pH}$, light, $\mathrm{O}_{2}$ and temperature (Hadjal, Dhuique-mayer, Madani, Dornier, \& Achir, 2013; Seyedrazi et al., 2011). In order to overcome the instability problems of these bioactive compounds, which results in restricted commercial applications, encapsulation has become an important tool, improving shelf life and biological effects. Moreover, encapsulation allows the controlled release of these functional pigments under desired conditions, for instance following oral administration (Santos \& Meireles, 2010).

Niosomes (non ionic surfactant vesicles) are vesicles delimited by close concentric bilayers obtained by the self assembly of non ionic surfactants, with or without incorporation of 
cholesterol, after hydration in aqueous media (Akhilesh, Bini, \& Kamath, 2012). These lamellar vesicles resemble liposomes in their structure and can be used as an attractive alternative to liposomes due to their high chemical stability and purity, content uniformity, affordable cost, convenient storage, and great variety of suitable surfactants (Shirsand, Para, Nagendrakumar, Kanani, \& Keerthy, 2012). Similarly to liposomes, niosomes have both hydrophilic and sandwiched lipophilic area to entrap both hydrophilic and hydrophobic substances, respectively (Solanki, Parikh, \& Parikh, 2007). As reported in the literature, niosomes can stabilize $\beta$-carotene against light, high temperatures and oxidative stress and allow its delivery to cultured cells at physiological concentrations (Socaciu, 2007). Despite the various reported advantages, niosomal dispersions present problems related to physical instability including vesicle fusion, aggregation, sedimentation and leakage during storage. Proniosomes, which are dry and free flowing powders, have been developed for overcoming these problems, typical of vesicular systems, while preserving composition and features (Aboelwafa, El-Setouhy, \& Elmeshad, 2010; Solanki et al., 2007). Proniosomes can be hydrated in aqueous media to form niosomes immediately before use or they can be administered as powder to form niosomal dispersions as they contact with body liquids. Despite the great potential of these systems, most applications are directed to medical and pharmaceutical sciences (Mokhtar, Sammour, Hammad, \& Megrab, 2008; Yasam, Jakki, Natarajan, \& Kuppusamy, 2014). Commonly used carriers such as maltodextrin (MD), lactose monohydrate (LA) and mannitol (MA) have been used successfully in proniosome preparation (Akhilesh, Faishal, \& Kamath, 2012). Pullulan (PU), an extracellular microbial polysaccharide, has been already used as a carrier for encapsulation of CTX (Hojjati, Razavi, Rezaei, \& Gilani, 
2013), turmeric (Kshirsagar, Yenge, Sarkar, \& Singhal, 2009) and beetroot pigment (Serris $\&$ Biliaderis, 2001). However, to the best of our knowledge, this is the first report on incorporation of CTX in proniosomes. Therefore, the first aim of the present work was to prepare proniosome powders using different carriers and freeze drying technique and characterize them for their physical properties and CTX stability. Secondly, this study was also aimed to evaluate the effect of these carriers on the properties and stability of the entrapped CTX and niosomal dispersions prepared after hydration.

\section{Material and methods}

\subsection{Chemicals}

D-glucose, peptone, yeast extract, agar, malt extract and span 60 were provided from Sigma-Aldrich Chemical Company (St. Louis, MO, USA). The CTX standard for HPLC analysis was purchased from Dr. Ehrenstorfer GmbH (Augsburg, Germany). Acetonitrile, dichloromethane, methanol (HPLC grade), lactose monohydrate (LA), cholesterol, absolute ethanol, sodium dihydrogen phosphate, disodium hydrogen phosphate, sodium chloride, potassium bromide $(\mathrm{KBr})$ and phosphotungstic acid were supplied from Merck (Darmstadt, Germany). Maltodextrin (MD) with a DE of 20 was procured from Dextroseiran Company (Tehran, Iran). Mannitol (MA) was obtained from Farmalabor (Milan, Italy) and pullulan (PU) was a product of Hayashibara Biochemical Laboratory (Okayama, Japan). Beetroot molasses were purchased from the Qazvin Sugar Industry (Qazvin, Iran). All other chemicals used in the experiments were of analytical grade. Ultrapure water (from a Millipore system) was used throughout the experiments. 


\subsection{Methods}

\subsubsection{CTX production}

The strain of bacterium D. natronolimnaea HS-1 (DSM 44860) used in this work was supplied by Bioprocess Engineering Laboratory (BPEL), University of Tehran, Iran. It was maintained on yeast/malt agar (YMA) plates containing glucose $(10 \mathrm{~g} / \mathrm{l})$, peptone $(5 \mathrm{~g} / \mathrm{l})$, yeast extract $(5 \mathrm{~g} / \mathrm{l})$, malt extract $(3 \mathrm{~g} / \mathrm{l})$ and agar $(15 \mathrm{~g} / \mathrm{l})$ at $4{ }^{\circ} \mathrm{C}$ and $\mathrm{pH} 7.31$. Single colonies were transferred to a fresh plate of YMA, incubated for 4 days at $28 \pm 1{ }^{\circ} \mathrm{C}$ and kept under refrigeration at $4{ }^{\circ} \mathrm{C}$. After inoculating with colonies of D. natronolimnaea $\mathrm{HS}$ 1, Erlenmeyer flask containing beetroot molasses (40 g/l) and yeast extract (10 g/l) were incubated in an orbital incubator (model Stuart S150; Staffordshire, UK) at $180 \mathrm{rpm}$ and $28 \pm 2{ }^{\circ} \mathrm{C}$ for 6 days to produce CTX (Gharibzahedi et al., 2013c, 2013d).

\subsubsection{Pigment extraction and analysis}

After the fermentation process, aliquots of cultures $(10 \mathrm{~mL})$ were centrifuged (Hettich, Tuttlingen, Germany) at $6000 \times \mathrm{g}\left(4^{\circ} \mathrm{C}\right)$ for $10 \mathrm{~min}$ and the cell pellets were washed twice with physiological water and centrifuged again. These cells were resuspended 3 times in absolute ethanol ( $3 \mathrm{~mL}$ ) by vortexing for $5 \mathrm{~min}$ and centrifuged again until pellets were colourless. A water bath $\left(45^{\circ} \mathrm{C}\right)$ was also used to enhance pigments extraction (Gharibzahedi, Razavi, \& Mousavi, 2013b). The ethanol extracts were filtered through a $0.2 \mu \mathrm{m}$ hydrophobic fluorophore membrane (Sigma-Aldrich Co., St. Louis, MO, USA). The absorbance of the ethanol extracts was measured at $\lambda$ max $=474 \mathrm{~nm}$ using a UV-visible spectrophotometer (PerkinElmer, Lamba25, USA) and total carotenoid (TC) concentration was calculated using the Eq. 1: 
$T C(\mu g / L)=\frac{A_{474} \times V_{S} \times 10^{9}}{A_{1}^{1 \%} \mathrm{~cm} \times 100} \quad$ Eq. 1

where $A_{474}, V s$ and $A_{1}^{1 \%} \mathrm{~cm}$ are the absorbance maximum of $T C$ in ethanol, the volume of sample solution, and the specific absorption coefficient of TC for a $1 \%$ solution in a $1 \mathrm{~cm}$ cell (in ethanol, $\left.A_{1}^{1 \%} \mathrm{~cm}=2200\right)$, respectively (Gharibzahedi et al., 2012).

Individual carotenoids were analyzed according to the method of Razavi et al. (2006) modified by Gharibzahedi et al. (2014) using a Knauer (Berlin, Germany) HPLC system on a Lichrospher $100 \mathrm{RP}-18$ silica column $(5.0 \mathrm{~mm}, 250 \mathrm{~mm} \times 4 \mathrm{~mm})$ at $35^{\circ} \mathrm{C}$. The isocratic mobile phase used was acetonitrile/methanol/dichloromethane solvent mixture (71:22:7, $\mathrm{v} / \mathrm{v} / \mathrm{v}$ ) at a flow rate of $2 \mathrm{~mL} / \mathrm{min}$. A precolumn of the same material was used as a guard column.

\subsubsection{Preparation of proniosome powders}

Four different carriers including MD, MA, LA and PU were employed to prepare formulations. Accurately weighed amounts of span $60(100 \mu \mathrm{mol})$, cholesterol $(100 \mu \mathrm{mol})$ and CTX (equivalent to $3 \mathrm{mg}$, considering that CTX in TC is 90.2\%) were dissolved completely in $2 \mathrm{~mL}$ ethanol and then added slowly to a water solution of the carrier (200 $\mathrm{mg}$ in $8 \mathrm{~mL}$ ) under magnetic stirring. This mixture was freeze dried (FDU-8606, Operon Co, Korea) to produce proniosome powder. The obtained proniosome powder was stored in a refrigerator at $4 \pm 1^{\circ} \mathrm{C}$. Unloaded proniosome powder (blank), without CTX, was prepared using the same method.

\subsubsection{Preparation of niosomes}


Niosomes were formed by hydration, combined with sonication, of the proniosome powder. An accurately weighed amount of proniosome powder was dissolved in $2 \mathrm{~mL}$ water to produce conventional niosomes. To reduce vesicular size and lamellarity, niosomes underwent probe sonication (Soniprep 150 plus, MSE Crowley, UK) at $13.1 \%$ amplification strength for 30 cycles with $5 \mathrm{~s}$ pulse on and pulse off intervals in an ice bath. Formulations were characterized after one day incubation. The obtained niosome dispersions were stored in a refrigerator at $4 \pm 1{ }^{\circ} \mathrm{C}$.

\subsubsection{Size analysis, size distribution and zeta potential measurement}

Mean size, size distribution and zeta potential of unloaded and CTX loaded niosomes were determined by dynamic light scattering (DLS) using a Malvern Nano ZS (Worchestershire, Malvern Instruments Ltd., UK) at $25^{\circ} \mathrm{C}$. The particle size distribution was measured as the polydispersity index (PDI). For the measurement, niosome formulations were diluted (1:10) with water to avoid multiple scattering effects and the vesicle diameter, PDI and zeta potential were determined. Niosomal dispersions were kept in airtight sealed glass vials at $4{ }^{\circ} \mathrm{C}$ and wrapped in aluminium foil to protect from light. Vesicles stability was measured after preparation and after 1 month.

\subsubsection{CTX recovery}

The CTX recovery of freeze dried proniosome powders was determined as follows. An accurately weighed amount of proniosome powder was dissolved in methanol and the resulting dispersion was centrifuged at $10000 \mathrm{rpm}$ for 1 hour. CTX was estimated 
spectrophotometrically at $474 \mathrm{~nm}$ and the CTX recovery (\%) was calculated according to the following equation (Eq. 2):

$C R(\%)=\frac{C_{0}}{C_{T}} \times 100 \quad$ Eq. 2

where CR is the CTX recovery; $\mathrm{C}_{0}=$ is the CTX of freeze dried powder; and $\mathrm{C}_{\mathrm{T}}$ is the CTX used for proniosomes preparation (before freeze drying).

\subsubsection{Entrapment efficiency}

Niosomal dispersions were evaluated for entrapment efficiency after preparation and 1 month on storage at $4{ }^{\circ} \mathrm{C}$. For determination of entrapment efficiency, the non entrapped CTX in niosomes was separated using the dialysis technique. $1 \mathrm{~mL}$ of niosomal suspension was pipetted into a dialysis tubing (Spectra/Por ${ }^{\circledR}$ membranes: $12-14 \mathrm{kDa}$ MW cut off, $3 \mathrm{~nm}$ pore size; Spectrum Laboratories Incorporation, USA) immersed in $1000 \mathrm{~mL}$ of water and magnetically stirred for 4 hours. Subsequently, $100 \mu \mathrm{L}$ of niosomal suspension was taken and $900 \mu \mathrm{L}$ of methanol was added to disrupt niosomes. The resulting solution was centrifuged at $10000 \mathrm{rpm}$ for 1 hour. CTX was estimated spectrophotometrically at $474 \mathrm{~nm}$ and the entrapment efficiency was expressed according to the following equation (Eq. 3):

$E E(\%)=\frac{C_{e}}{C_{0}} \times 100 \quad$ Eq. 3

where EE is the entrapment efficiency; $\mathrm{C}_{\mathrm{e}}$ is the CTX entrapped into niosomes; and $\mathrm{C}_{0}$ is the CTX of freeze dried powder.

\subsubsection{Morphological observations}

\subsubsection{Niosome}


Proniosome powder was placed on a glass slide without a cover slip and a drop of water was added to the particles. Evaluation of vesicle formation and shape of niosomes during hydration of proniosome powder was performed using an ordinary light microscope (Nicon, HFX-DX, Japan). Optimized formula was also observed and photographed with a Philips CM120 Transmission Electron Microscope (FEI Corporation, Eindhoven, The Netherlands) at an accelerating voltage of $80 \mathrm{kV}$ after negative staining using $1 \%$ aqueous phosphotungstic acid.

\subsubsection{Proniosome powder}

Surface morphology of proniosome powders was studied using a Hitachi S-4800 (Monocomp, Madrid, Spain) scanning electron microscope (SEM) at $5 \mathrm{kV}$ scanning electron microscopy. A small amount of powder was placed on a stud and then coated with a thin layer of gold to make them electrically conductive. The coated samples were analyzed for surface morphology and photographed.

\subsubsection{Micromeritics properties of proniosome powders}

The flow properties were studied by measuring the angle of repose, Carr's compressibility index and Hausner's ratio. The angle of repose of proniosome powders was measured by using fixed funnel method. Briefly, the proniosome powder was poured into a funnel which was fixed at a position so that the outlet orifice of the funnel was $5 \mathrm{~cm}$ above a black surface. The powder flows down from the outlet orifice to form a cone on the surface and the angle of repose was then calculated by measuring the height of the cone (h) and the 
radius of its base (r) with the help of calibrated scale (Patil-Gadhe \& Pokharkar, 2014). The angle of repose $(\theta)$ was calculated as Eq. 4.

$\theta=\tan ^{-1}\left(\frac{\mathrm{h}}{\mathrm{r}}\right)$

A known mass of proniosome powder was poured into a calibrated measuring cylinder and the volume occupied by this powder was recorded. The tapped density was determined by volume measurement of the tapped mass until no further changes in the powder volume were observed (Shah, Tawakkul, \& Khan, 2008). The Carr's compressibility index (Carr, 1965) and Hausner's ratio (Hausner, 1967) were calculated according to Eq. 5 and Eq. 6:

$C I(\%)=\frac{\rho_{\text {tap }}-\rho_{\text {bulk }}}{\rho_{\text {tap }}} \times 100 \quad$ Eq. 5

$H R=\frac{\rho_{\text {tap }}}{\rho_{\text {bulk }}} \quad$ Eq. 6

where CI is Carr's compressibility index; HR is Hausner's ratio; $\rho_{\text {tap }}$ is the tap density; and $\rho_{\text {bulk }}$ is the bulk density.

\subsubsection{Fourier transform infrared spectroscopy (FTIR)}

The FTIR spectra of CTX, optimized sample, physical mixture and unloaded formulation of the optimized proniosome powder were collected using Perkin Elmer FTIR Spectrometer Spectrum One (PerkinElmer Incorporation, Buckinghamshire, UK) in the wavelength region of $4000-450 \mathrm{~cm}^{-1}$. For preparation of the physical mixture, accurately weighed amounts of optimized sample ingredients were just mixed together before analysis. The procedure consisted of mixing a sample in a mortar with $\operatorname{KBr}(1: 100)$ and compressing into small tablet by applying a pressure of 14 tons in a hydraulic press. The tablet was placed in the infrared beam path and the spectrum was recorded. 


\subsubsection{Differential scanning calorimetry (DSC)}

DSC analysis was used to evaluate the thermal behaviour of CTX, optimized sample, physical mixture and unloaded formulation of the optimized proniosome powder. The DSC curves of different sample were recorded on a Perkin Elmer DSC 6 (Perkin Elmer Corporation, Norwalk, CT, USA) differential scanning calorimeter. The samples were heated in aluminium crimped pans under nitrogen flow at the heating rate of $10{ }^{\circ} \mathrm{C} / \mathrm{min}$ from $50-350{ }^{\circ} \mathrm{C}$.

\subsubsection{Powder X-ray diffraction (PXRD)}

PXRD of CTX, optimized sample, physical mixture and unloaded formulation of optimized proniosome powder were conducted using a Rigaku Miniflex diffractometer (Rigaku

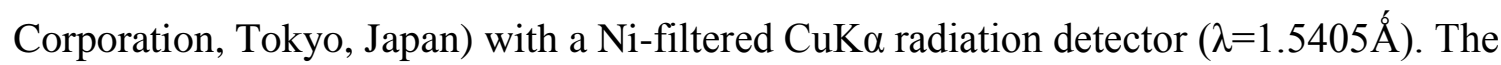
powder sample was spread on glass sample holder and its surface was pressed and smoothed with a glass slide. The voltage and current generator were set up at $30 \mathrm{kV}$ and 35 $\mathrm{mA}$ respectively. All samples were analyzed in the $2 \theta$ range from $3^{\circ}$ to $60^{\circ}$ with a scan angular speed of $2 \%$ min and a scan step time of $2.00 \mathrm{~s}$.

\subsubsection{Stability studies}

\subsubsection{Effect of storage and temperature}

Proniosome powders were stored in airtight sealed glass vials at two different temperatures (4 and $25^{\circ} \mathrm{C}$ ). Vials were wrapped in aluminium foil to protect samples from light. The 
proniosome powders were tested after preparation and after 1 month for CTX recovery by the spectrophotometer.

\subsubsection{Effect of light}

Proniosome powders were stored in airtight sealed glass vials at temperature controlled incubators $\left(25^{\circ} \mathrm{C}\right)$ under light condition for 15 days. The intensity of 2000 lx was used with cool white and fluorescent lamps (30 cm away from the samples). Proniosome powder was analyzed for CTX recovery at regular intervals of time (0, 2, 5, 10 and 15 days).

\subsubsection{Statistical analysis}

Data are expressed as mean \pm standard deviation $(n=3$ independent samples). Analysis of the data was made using ANOVA from SAS statistical software (version 9.0). Comparison among the means was carried out by Duncan's multiple range test at $\mathrm{p}<0.05$ as a minimal level of significance. Statistical analysis between loaded and unloaded powders was performed by Students' t-test at $\mathrm{p}<0.05$.

\section{Results and discussion}

\subsection{Preliminary investigation}

D. natronolimnaea HS-1 produced orange red pigments which were extracted by ethanol. The HPLC analysis of the ethanol extract showed that CTX represented $90.20 \pm 0.10 \%$ of the mixture (Supplementary Table 1). This result is in agreement with that obtained by Gharibzahedi et al. (2013c). 
The extracted CTX was used to prepare proniosomes, provesicular powders that provide a simple way of administering low bioavailable compounds by taking advantages of the transporter properties of the niosomes but avoiding the instability phenomena classically shown by the hydrated vesicles

Results obtained here showed that niosomes prepared with span 60/cholesterol (1:1 $\mathrm{mol} / \mathrm{mol})$ were small in size $(\leq 200 \mathrm{~nm})$ and quite homogeneously dispersed $(\mathrm{PDI} \leq 0.3)$. No micelles could be detected by TEM and DLS analyses. As shown in the literature, Span 60 is one of the most used surfactants for niosome production because of its low HLB value (4.7) and the critical packing parameter between 0.5 and 1 (Aboelwafa et al., 2010; Akhilesh, Bini, et al., 2012; Marianecci et al., 2014).

In the first step of the study, conventional methods for proniosome preparation, such as hand shaking method, slurry method and slow spray coating method (Radha, G. V., Rani, T. S., \& Sarvani, 2013), were used. However, they were not suitable for obtaining CTX encapsulation. Therefore, in this work, freeze drying was employed in proniosome production. After a preliminary screening study in which several different coating materials were tested, we selected type and amount of carriers able to produce both proniomal powders with suitable flow properties and, after hydration, CTX entrapping niosomes.

\subsection{Size analysis, size distribution and zeta potential measurement}

As can be seen in Table 1, results of the current study showed that the niosomes produced by hydration and sonication of the proniosomes were small in size $(\leq 200 \mathrm{~nm})$ and quite homogeneously dispersed ( $\mathrm{PDI} \leq 0.3)$. However, the type of carrier affected both mean vesicle size and size distribution, although unloaded vesicles showed a similar mean size 
(171-179 nm), except for unloaded PUPP having the smallest vesicles (130 nm).

Nevertheless, the difference in size between each unloaded and CTX loaded pair of niosomes were not statistically significant ( $p>0.05)$. This is indicative of a good incorporation of CTX in the lamellar membrane of the hydrated niosomes. One of the hypotheses regarding efficient niosomes formation from proniosomes is based on the dissolution property of the carrier to facilitate hydration of the surfactant. In this study two low molecular weight (MW) compounds (MA, LA), one oligosaccharide (MD) and a polysaccharide were used as carriers. As shown in Table 1, the two low MW carriers led to similar vesicle size (in the range 175-183 nm) while the oligosaccharide MD gave the largest niosomes $(207 \pm 28 \mathrm{~nm})$ and the polymer the smallest and most homogeneous ones (mean size $=141 \pm 18 \mathrm{~nm}, \mathrm{PDI}=0.15 \pm 0.05$ ). This behaviour is probably due to the different solubility and viscosity of the carriers. On storage, the size of LAPP and PUPP niosomes remained constant while MAPP niosomes showed only a 10\% increase and the MDPP niosomes underwent the highest enlargement ( 60\%).

Zeta potential is an important parameter for evaluating the surface charge of niosomes and, consequently, their stability against aggregation and fusion (Sezgin-Bayindir, Antep, \& Yuksel, 2015). Zeta potential of niosomes produced by hydration and sonication was always highly negative but the incorporation of CTX even improved the negative charge. However, only MAPP niosomes had a significantly higher negative zeta potential than the unloaded MAPP niosomes ( $<<0.05$, Table 1). The zeta potential of the unloaded niosomes ranged between $-57.5 \pm 4.2 \mathrm{mV}$ (unloaded LAPP) to $-72.5 \pm 3.4 \mathrm{mV}$ (unloaded PUPP) while the CTX loaded niosomes showed a zeta potential value from $-74.8 \pm 4.0 \mathrm{mV}$ (MAPP) to 61.2 \pm 3.9 (LAPP). In particular, the MAPP niosomes showed the highest negative zeta 
potential $(-74.8 \pm 4.0 \mathrm{mV})$ after preparation and sonication as well as on storage. As shown in Table 1, our results revealed that the type of carrier used in proniosome formulation and storage affected zeta potential significantly $(\mathrm{p}<0.05)$. Niosomes of MAPP showed the highest negative zeta potential after preparation and during storage, thus, indicating the high stability of the obtained niosomes.

Overall results, indeed surprising since all the formulation components are non ionic, might depend on the adsorption of ions dissolved in the aqueous medium or be the consequence of the interactions between the dispersion medium and the free electron pairs of these formulation materials, although all non ionic molecules.

Finally, as can be seen from the $\mathrm{p}$ values, type of carrier and storage for 30 days had a significant interaction on vesicle size and PDI of niosomal dispersion $(\mathrm{p}<0.05)$.

\subsection{Entrapment efficiency}

The entrapment efficiency is one of the most important parameters in the evaluation of niosomal formulations (Aboelwafa et al., 2010). It is expressed as the ability of a formulation to retain expected molecules in the bilayer membrane or in the aqueous core of the vesicles (Tavano, de Cindio, Picci, Ioele, \& Muzzalupo, 2014). The entrapment efficiency of niosomes produced after hydration and sonication of the proniosome powders is reported in Table 1. The results demonstrate that the type of carrier used for proniosome preparation affected entrapment efficiency significantly $(\mathrm{p}<0.05)$.The encapsulation efficiency of CTX in formulations varied between $55.3 \pm 1.8 \%$ and $74.1 \pm 2.7 \%$ after hydration and sonication. Among these formulations, niosomes of MAPP (74.1\%) showed the highest entrapment efficiency followed by niosomes of MDPP (67.2\%), LAPP (63.9\%) 
and PUPP (55.3\%). Additionally, niosomes of MAPP stabilized CTX well in bilayers during 30 days of storage: no CTX leakage was observed at the end of the stability study, while the other formulations showed a reduced loaded CTX. In particular, the leakage increased from MDPP ( 5\%) to PUPP $(\sim 7.5 \%)$ to LAPP ( 12\%). This result is in accordance with that published by Ahuja \& Saroha (2014) who applied MA for proniosome preparation with higher entrapment efficiency than MD. According to the obtained results, the MAPP formulation was chosen for further studies as a consequence of the highest zeta potential value and entrapment efficiency, and the absence of CTX leakage, which are predictive of great stability.

\subsection{Morphological observations}

\subsubsection{Niosome}

As described in the experimental section, formation of niosomes by hydration of proniosomes was demonstrated by following the conversion of proniosomes to vesicles after addition of a drop of water under an optical microscope. In these conditions the production of vesicles was progressive and fast but it produced large multilamellar vesicles (mean diameter $>6 \mu \mathrm{m}$ ), as can be seen in Figure 1a where niosomes produced from MAPP have a thick membrane, indicative of multilamellar vesicles. Indeed, the optical microscope cannot give a good image of the vesicles but it is well known that multilamellar vesicles are always at first produced by hydrating proniosomes as well as phospholipids. Figure $1 \mathrm{~b}$ shows instead the vesicles obtained by hydration and sonication of the same formulation MAPP. The sonication led to spherical, small unilamellar vesicle, whose size well correlated with that obtained by DLS $(200 \mathrm{~nm})$. 


\subsubsection{Proniosome powder}

Scanning electron microscope is a system for image viewing which employs electrons to study surface morphology such as roundness, smoothness and the size distribution of proniosome powders (Gupta et al., 2014; Omer, 2014). SEM images of MDPP, MAPP, LAPP and PUPP are illustrated in Figure 2. MDPP produced small spherical particles with rough surfaces and size distribution in the range 1.12-2.62 $\mu \mathrm{m}$ (Figure 2a) which may be due to the highly porous surface of MD as a carrier (Cheriyan et al., 2015; Solanki, Parikha, \& Parikh, 2008). In contrast, PUPP had spherical particles with smooth surfaces and broad size distribution (0.28-2.92 $\mu \mathrm{m})$ (Figure 2d). MAPP particles were long with broad size distribution, irregular in shape but with smooth surfaces (Figure 2b). The long and thin particles of MAPP would be indicative of a high surface area of the carrier and predictive of a rapid and easy hydration (Blazek-Welsh \& Rhodes, 2001). LAPP had rough and irregular crystal shapes coated with other ingredients of proniosome powder (Figure 2c). This is in agreement with findings reported by Abd-Elbary et al. (2008) who found that fine structures as whiskers and sharp corners of LA appears to be smoother after proniosome preparation because of the filling effect of surfactant and brief dissolution of surface molecules of LA. In conclusion, as shown, the nature of the carrier deeply affected the morphology of the powders and, consequently, the properties of the hydrated vesicles.

\subsection{Micromeritics properties}

The flow properties of the powder formulations are important parameters from industrial view point because improper flowability may lead to problems in storage, handling and 
processing operations. The micromeritics properties were assessed by determining angle of repose ( $\theta$ ), Carr's index (CI) and Hausner's ratio (HR) and the results are summarized in Table 2. The angle of repose is defined as the angle formed by the horizontal base of the bench surface and the edge of a cone-like pile of the powder. The compressibility of the proniosome powder is stated as Carr's index and Hausner ratio. The angle of repose for all the proniosome powders prepared was found to be in the range of $25-34^{\circ}$ whereas Carr's index and Hausner ratio were in the range of $21-35 \%$ and $1.27-1.53$, respectively. The type of carrier affected significantly ( $\mathrm{p}<0.05)$ the angle of repose, Carr's index and Hausner's ratio values, although variations were very small (Table 2). The angle of repose less than $30^{\circ}$ indicates excellent flow properties. Particles with high internal friction or cohesion increase the value of angle of repose. Moreover, Carr's index and Hausner's ratio should be less than $20 \%$ and 1.25 , respectively, to ensure optimal flowability for proniosome powders (Singh, Vuddanda, Singh, \& Srivastava, 2013). MDPP showed the lowest angle of repose $\left(25 \pm 3^{\circ}\right)$, Carr's index $(21 \pm 4 \%)$ and Hausner's ratio $(1.27 \pm 0.07)$ providing the best micromeritics properties among all. This is consistent with the results obtained by SEM analysis for proniosome powders (Sec 3.), in which it was observed that MDPP had round and uniform spherical particles, which can offer better flow properties. Therefore, in general, other excipients should be added to the freeze dried proniosomes to optimize their flowability.

\subsection{FTIR}

FTIR is one of the most practical methods for investigation of molecular structures and functional groups (Bai et al., 2011). One application of this technique is the evaluation of 
selected substance-excipient compatibility, which is a prerequisite for the design of a formulation. In this study, with the aim to explore the possible interactions between CTX and pronoisomes, FTIR spectra of CTX, physical mixture and unloaded MAPP (without CTX) were compared to MAPP. As shown in supplementary Figure 2 the pure CTX exhibited characteristic peaks at $3435 \mathrm{~cm}^{-1}(\mathrm{OH}), 3023-2866 \mathrm{~cm}^{-1}$ (H-Csp3 and H-Csp2), $1654 \mathrm{~cm}^{-1}$ (conjugated CO), $1606 \mathrm{~cm}^{-1}$ and $1557 \mathrm{~cm}^{-1}(\mathrm{C}=\mathrm{C}), 1399 \mathrm{~cm}^{-1}$ and $1364 \mathrm{~cm}^{-1}$ (gem-dimetyl), $1092 \mathrm{~cm}^{-1}$ and $1032 \mathrm{~cm}^{-1}(\mathrm{OH})$ and $975 \mathrm{~cm}^{-1}(\mathrm{CH}=\mathrm{CH}$, trans)

(Supplementary Figure 2a). The characteristic CTX peaks were also found in the physical mixture spectrum (Supplementary Figure 2d), thus indicating that any significant interaction between CTX and the excipients occurred. Because of its small amount, CTX stretchings were not visible in the MAPP spectrum (Supplementary Figure 2c), which was very similar to that of the unloaded MAPP (Supplementary Figure 2b). Nevertheless, the mild differences in bands intensity and the presence of remarkably diffused peaks in the MAPP proniosome spectra with respect to that of the physical mixture, indicated a physical interaction between CTX and formulation components.

\subsection{DSC}

The DSC thermograms of CTX, MAPP, physical mixture of MAPP components and unloaded MAPP are shown in Supplementary Figure 3. As CTX exhibited a melting point at an onset temperature of $216.28^{\circ} \mathrm{C}$ (Supplementary Figure 3a), the analyses revealed that endothermic peak of CTX is not detectable neither in the physical mixture nor in the MAPP. In the physical mixture thermogram (Supplementary Figure 3c) span 60 and other ingredients were located at an onset temperature of 54.70 and $164.10^{\circ} \mathrm{C}$, respectively. Although the main transition temperature $\left(\mathrm{T}_{\mathrm{m}}\right)$ 
was similar for all thermograms, the total integrated zone below the thermogram peak $(\Delta H)$ varied among formulations. The physical mixture had the highest $\Delta \mathrm{H}(235.49 \mathrm{~J} / \mathrm{g})$ followed by unloaded MAPP (198.25 J/g), MAPP (183.96 J/g) and CTX (178.99 J/g). $\Delta \mathrm{H}$ has direct effect on crystallinity; the higher the $\Delta \mathrm{H}$, the more crystallinity. In this respect, preparation of proniosome powder and inclusion of CTX decreased crystallinity.

\subsection{PXRD}

The PXRD patterns of CTX, MAPP, physical mixture and unloaded MAPP are represented in Supplementary Figure 4. The physical mixture showed numerous high intensity diffraction peaks demonstrating its crystalline nature probably due to the crystallinity of the main component (mannitol) (Supplementary Figure 4a). The unloaded MAPP diffractogram indicated that the crystalline character of physical mixture changed during the preparation process as the intensity of some peaks altered and new diffraction peaks appeared (Supplementary Figure 4d). Even if it was believed that the amount of CTX was too low to be detected, it affected the PXRD pattern of MAPP (Supplementary Figure 4c). The presence of CTX in preparation of proniosome powder not only reduced the intensity of peaks even more but also changed the pattern of some of them. The results were further confirmed by DSC analysis wherein the presence of CTX decreased crystallinity (Sec 3.8).

\subsection{Stability studies}

\subsubsection{Effect of storage and temperature}

Thermal treatment to CTX in the presence of oxygen results in formation of oxidation products as well as isomers. Physical stability of proniosome powders was carried out in 
term of CTX recovery at two different temperatures $\left(4\right.$ and $\left.25^{\circ} \mathrm{C}\right)$. The CTX recovery was determined after preparation and after 30 days as shown in Figure3. There was no significant interaction between storage temperature and type of carrier on CTX recovery $(\mathrm{p}>0.05)$. It was observed that as the storage temperature increased, CTX recovery decreased significantly $(\mathrm{p}<0.05)$. Similar results were also obtained by Hojjati et al. (2014) who reported the deterioration of microencapsulated CTX was accelerated by increasing the incubation temperature of storage. The stability of CTX in proniosome powder was higher than CTX in microencapsulated powder prepared by Hojjati et al. (2014).

\subsubsection{Effect of Light}

As all the carotenoid compounds, CTX reacts with different types of radicals by different mechanisms of electron transfer that lead to the formation of radical cations or radical adducts. Besides attack by radicals, CTX is also degraded by light to form cis-trans isomers. Figure 4 shows the recovery of CTX in MAPP at room temperature $\left(25^{\circ} \mathrm{C}\right)$ in the light. The amount of CTX which can be recovered from proniosome powder was determined after $0,2,5,10$ and 15 days. The light showed a significant effect $(\mathrm{P}<0.05)$ on the CTX recovery of MAPP at $25^{\circ} \mathrm{C}$, which is in good agreement with Palozza et al. (2006) who investigated the stability of $\beta$-carotenes in niosomes at room temperature $\left(25^{\circ} \mathrm{C}\right)$ in the light and showed that the carotenoids are much more consumed in the light than in the dark because of sensitive double bonds existed in the chain of carotenoids.

\section{Conclusion}


Carotenoids such as CTX are colourful and sensitive pigments found in different sources.

Proniosomes are water soluble powders with great stability and industrial scalability. They can provide convenient, nontoxic and inexpensive vehicles for dissolving and stabilizing CTX in functional food products. According to the obtained results, CTX can be incorporated and well stabilized in proniosome powders prepared with MA as carrier. Although light and high temperatures $\left(45^{\circ} \mathrm{C}\right)$ were drastic factors for the CTX stability, incorporation in proniosomes retarded its degradation. The findings of the current study on proniosomes highlight a higher CTX stability especially at temperatures higher than the room temperature. However, further studies should be performed to assess the ability of different carriers and their mixtures for formulation of proniosomes with better properties. Stability studies according to ICH and WHO guidelines should also be performed in a more advanced step of the study.

\section{Acknowledgement}

The authors would like to extend their sincere appreciation for the technical support provided by the University of Cagliari and University of Milan. This work was financially supported by the University of Tehran and Iranian center of excellence for application of modern technologies for producing functional foods and drinks. We thank also Iran National Science Foundation for funding the above work.

\section{References}

Abd-Elbary, A., El-laithy, H. M., \& Tadros, M. I. (2008). Sucrose stearate-based proniosome-derived niosomes for the nebulisable delivery of cromolyn sodium. 
International Journal of Pharmaceutics, 357(1-2), 189-198. http://doi.org/10.1016/j.jpharm.2008.01.056

Aboelwafa, A. A., El-Setouhy, D. A., \& Elmeshad, A. N. (2010). Comparative study on the effects of some polyoxyethylene alkyl ether and sorbitan fatty acid ester surfactants on the performance of transdermal carvedilol proniosomal gel using experimental design. AAPS PharmSciTech, 11(4), 1591-1602. http://doi.org/10.1208/s12249-010-9539-0

Ahuja, M., \& Saroha, K. (2014). Proniosomes formulations using maltodextrin and mannitol as carriers. International Journal of Pharmaceutical and Chemical Sciences, $3(2), 547-551$.

Akhilesh, D., Bini, K. B., \& Kamath, J. V. (2012). Review on span-60 based non-ionic surfactant vesicles ( niosomes ) as novel drug delivery. International Journal of Research in Pharmaceutical and Biomedical Sciences, 3(1), 6-12.

Akhilesh, D., Faishal, G., \& Kamath, J. V. (2012). Comparative study of carriers used in proniosomes. International Journal of Pharmaceutical and Chemical Sciences, 1(1), $164-173$.

Bai, C., Peng, H., Xiong, H., Liu, Y., Zhao, L., \& Xiao, X. (2011). Carboxymethylchitosancoated proliposomes containing coix seed oil: Characterisation, stability and in vitro release evaluation. Food Chemistry, 129(4), 1695-1702. http://doi.org/http://dx.doi.org/10.1016/j.foodchem.2011.06.033

Blazek-Welsh, A. I., \& Rhodes, D. G. (2001). Maltodextrin-based proniosomes. AAPS pharmSci, 3(1), E1.

Carr, R. L. (1965). Evaluating flow properties of solids. Chemical Engineering, 72, 69-72.

Cheriyan, P., George, B. J., Thomas, N., Raj, P., Samuel, J., \& Carla, S. B. (2015). 
Formulation and characterization of maltodextrin based proniosomes of cephalosporins. World Journal of Pharmaceutical Sciences, 3(1), 62-74.

Clark, T. H., Faustman, C., Chan, W. K. M., Furr, H. C., \& Riesen, J. W. (1999). Canthaxanthin as an antioxidant in a liposome model system and in minced patties from rainbow trout. Journal of Food Science, 64(6), 982-986.

Ekin Gumus, C., Davidov-pardo, G., \& Julian McClements, D. (2016). Food Hydrocolloids Lutein-enriched emulsion-based delivery systems : Impact of Maillard conjugation on physicochemical stability and gastrointestinal fate. Food Hydrocolloids, 60, 38-49. http://doi.org/10.1016/j.foodhyd.2016.03.021

Gharibzahedi, S. M. T., Razavi, S. H., \& Mousavi, M. (2013a). Kinetic analysis and mathematical modeling of cell growth and canthaxanthin biosynthesis by Dietzia natronolimnaea HS-1 on waste molasses hydrolysate. RSC Advances, 3(45), 2349523502. http://doi.org/10.1039/C3RA44663H

Gharibzahedi, S. M. T., Razavi, S. H., \& Mousavi, S. M. (2013b). Comparison of antioxidant and free radical scavenging activities of biocolorant synthesized by Dietzia natronolimnaea HS-1 cells grown in batch, fed-batch and continuous cultures. Industrial Crops and Products, 49, 10-16. http://doi.org/http://dx.doi.org/10.1016/j.indcrop.2013.03.019

Gharibzahedi, S. M. T., Razavi, S. H., \& Mousavi, S. M. (2013c). Psyllium husk gum: an attractive carbohydrate biopolymer for the production of stable canthaxanthin emulsions. $\quad$ Carbohydrate Polymers, 92(2), 2002-2011. http://doi.org/10.1016/j.carbpol.2012.11.083

Gharibzahedi, S. M. T., Razavi, S. H., \& Mousavi, S. M. (2013d). Ultrasound-assisted 
formation of the canthaxanthin emulsions stabilized by arabic and xanthan gums. Carbohydrate Polymers, 96(1), 21-30. http://doi.org/10.1016/j.carbpol.2013.03.085

Gharibzahedi, S. M. T., Razavi, S. H., \& Mousavi, S. M. (2014). Characterizing the natural canthaxanthin/2-hydroxypropyl-beta-cyclodextrin inclusion complex. Carbohydrate Polymers, 101, 1147-1153. http://doi.org/10.1016/j.carbpol.2013.10.074

Gharibzahedi, S. M. T., Razavi, S. H., Mousavi, S. M., \& Moayedi, V. (2012). High efficiency canthaxanthin production by a novel mutant isolated from Dietzia natronolimnaea HS-1 using central composite design analysis. Industrial Crops and Products, 40, 345-354. http://doi.org/http://dx.doi.org/10.1016/j.indcrop.2012.03.030

Gupta, R., Kumar, S., Gupta, N., Kumar, V., Prajapati, S. K., \& Kumar, A. (2014). The proniosomes development and optimization as a surrogated drug carrier for oral delivery of gliclazide. an-overview. World Journal of Pharmacy and Pharmaceutical Sciences, 3(9), 275-294.

Hadjal, T., Dhuique-mayer, C., Madani, K., Dornier, M., \& Achir, N. (2013). Thermal degradation kinetics of xanthophylls from blood orange in model and real food $\begin{array}{llll}\text { systems. } & \text { Food } & \text { Chemistry, } & \text { 138(4), }\end{array}$ http://doi.org/10.1016/j.foodchem.2012.12.022

Hausner, H. H. (1967). Friction conditions in a mass of metal powder. International Journal of Powder Metallurgy, 3, 7-13.

Hojjati, M., Razavi, S. H., Rezaei, K., \& Gilani, K. (2011). Spray drying microencapsulation of natural canthaxantin using soluble soybean polysaccharide as a carrier. Food Science and Biotechnology, 20(1), 63-69. http://doi.org/10.1007/s10068011-0009-6 
Hojjati, M., Razavi, S. H., Rezaei, K., \& Gilani, K. (2013). Effect of wall components on characteristics of natural canthaxanthin microencapsulated using spray drying. Iranian Journal of Nutrition Sciences and Food Technology, 8(3), 45-54.

Hojjati, M., Razavi, S. H., Rezaei, K., \& Gilani, K. (2014). Stabilization of canthaxanthin produced by Dietzia natronolimnaea HS-1 with spray drying microencapsulation. Journal of Food Science and Technology, 51(9), 2134-2140. http://doi.org/10.1007/s13197-012-0713-0

Kshirsagar, A. C., Yenge, V. B., Sarkar, A., \& Singhal, R. S. (2009). Efficacy of pullulan in emulsification of turmeric oleoresin and its subsequent microencapsulation. Food Chemistry, 113(4), 1139-1145. http://doi.org/http://dx.doi.org/10.1016/j.foodchem.2008.09.002

Marianecci, C., Di Marzio, L., Rinaldi, F., Celia, C., Paolino, D., Alhaique, F., .. Carafa, M. (2014). Niosomes from 80s to present: The state of the art. Advances in Colloid and Interface $\quad$ Science, $\quad 205, \quad 187-206$. http://doi.org/http://dx.doi.org/10.1016/j.cis.2013.11.018

Mokhtar, M., Sammour, O. A., Hammad, M. A., \& Megrab, N. A. (2008). Effect of some formulation parameters on flurbiprofen encapsulation and release rates of niosomes prepared from proniosomes. International Journal of Pharmaceutics, 361, 104-111. http://doi.org/10.1016/j.ijpharm.2008.05.031

Nasri Nasrabadi, M. R., \& Razavi, S. H. (2010). Enhancement of canthaxanthin production from Dietzia natronolimnaea HS-1 in a fed-batch process using trace elements and statistical methods. Brazilian Journal of Chemical Engineering, 27(4), 517-529. http://doi.org/10.1590/S0104-66322010000400003 
Omer, H. K. (2014). Spray-dried bioadhesive formulations for pulmonary delivery. University of Central Lancashire.

Palozza, P., Muzzalupo, R., Trombino, S., Valdannini, A., \& Picci, N. (2006). Solubilization and stabilization of beta-carotene in niosomes: delivery to cultured cells. Chemistry and Physics of Lipids, 139(1), 32-42. http://doi.org/10.1016/j.chemphyslip.2005.09.004

Patil-Gadhe, A., \& Pokharkar, V. (2014). Single step spray drying method to develop proliposomes for inhalation: a systematic study based on quality by design approach. Pulmonary Pharmacology \& Therapeutics, 27(2), 197-207. http://doi.org/10.1016/j.pupt.2013.07.006

Radha, G. V., Rani, T. S., \& Sarvani, B. (2013). A review on proniosomal drug delivery system for targeted drug action. Journal of Basic and Clinical Pharmacy, 4(2), 42-48. http://doi.org/10.4103/0976-0105.113609

Razavi, S. H., Blanchard, F., \& Marc, I. (2006). UV-HPLC/APCI MS method for separation and identification of the carotenoids produced by Sporobolomyces ruberrimus H110. Iranian Journal of Chemistry and Chemical Engineering, 25, 1-10.

Santos, D. T., \& Meireles, M. A. A. (2010). Carotenoid pigments encapsulation: fundamentals, techniques and recent trends. The Open Chemical Engineering Journal, 4, 42-50. http://doi.org/10.2174/1874123101004010042

Serris, G. S., \& Biliaderis, C. G. (2001). Degradation kinetics of beetroot pigment encapsulated in polymeric matrices. Journal of the Science of Food and Agriculture, 81(8), 691-700. http://doi.org/10.1002/jsfa.864

Seyedrazi, N., Razavi, S. H., \& Emam-Djomeh, Z. (2011). Effect of different pH on 
canthaxanthin degradation. World Academy of Science, Engineering and Technology, $5(11), 653-657$.

Sezgin-Bayindir, Z., Antep, M. N., \& Yuksel, N. (2015). Development and characterization of mixed niosomes for oral delivery using candesartan cilexetil as a model poorly water-soluble drug. AAPS PharmSciTech, 16(1), 108-117. http://doi.org/10.1208/s12249-014-0213-9

Shah, R. B., Tawakkul, M. A., \& Khan, M. A. (2008). Comparative evaluation of flow for pharmaceutical powders and granules. AAPS PharmSciTech, 9(1), 250-258. http://doi.org/10.1208/s12249-008-9046-8

Shirsand, S. B., Para, M. S., Nagendrakumar, D., Kanani, K. M., \& Keerthy, D. (2012). Formulation and evaluation of Ketoconazole niosomal gel drug delivery system. International Journal of Pharmaceutical Investigation, 2(4), 201-207. http://doi.org/10.4103/2230-973X.107002

Singh, S. K., Vuddanda, P. R., Singh, S., \& Srivastava, A. K. (2013). A Comparison between Use of Spray and Freeze Drying Techniques for Preparation of Solid SelfMicroemulsifying Formulation of Valsartan and In Vitro and In Vivo Evaluation. BioMed Research International, 2013, 1-13.

Socaciu, C. (2007). New technologies to synthesize, extract and encapsulate natural food colorants. Bulletin of University of Agricultural Sciences and Veterinary Medicine Cluj-Napoca. Animal Science and Biotechnologies, 64(1-2), 63-64.

Solanki, A., Parikh, J. R., \& Parikh, R. H. (2007). Formulation and optimization of piroxicam proniosomes by 3-factor, 3-level Box-Behnken design. AAPS PharmSciTech, 8(4), 86-93. http://doi.org/10.1208/pt0804086 
Solanki, A., Parikha, J., \& Parikh, R. (2008). Preparation, characterization, optimization, and stability Studies of aceclofenac proniosomes. Iranian Journal of Pharmaceutical Research, 7(4), 237-246.

Tanaka, T., Shnimizu, M., \& Moriwaki, H. (2012). Cancer chemoprevention by carotenoids. Molecules, 17(3), 3202-3242. http://doi.org/10.3390/molecules17033202

Tavano, L., de Cindio, B., Picci, N., Ioele, G., \& Muzzalupo, R. (2014). Drug compartmentalization as strategy to improve the physico-chemical properties of diclofenac sodium loaded niosomes for topical applications. Biomedical Microdevices, 16(6), 851-858. http://doi.org/10.1007/s10544-014-9889-6

Yasam, V. R., Jakki, S. L., Natarajan, J., \& Kuppusamy, G. (2014). A review on novel vesicular drug delivery: proniosomes. Drug Delivery, 21(4), 243-249. http://doi.org/10.3109/10717544.2013.841783 


\section{Figure legends}

Figure 1 Microphotographs showing formation of niosomes on proniosome powder after hydration with water (a) and TEM image of niosome dispersion after hydration and sonication of MAPP (b).

Figure 2 SEM micrographs of proniosome powders produced with various carriers MDPP (a); MAPP (b); LAPP (c); PUPP (d)

Figure 3 Changes in CTX recovery (\%) of four different formulations over 30 days of storage at 4 and $25^{\circ} \mathrm{C}(\mathrm{n}=3)$. No significant interaction was found between storage temperature and type of carrier on CTX recovery $(\mathrm{P}>0.05)$

Figure 4 Changes in the CTX recovery (\%) of MAPP over 15 days on storage in light. Means with different letters show a significant difference at $\mathrm{P}<0.05(\mathrm{n}=3)$ 
Table 1 Vesicle size, PDI, zeta potential and entrapment efficiency of niosomes prepared with different proniosome powders ${ }^{1,2}$

$\begin{array}{llll}\text { Mean size (nm) } & \text { PDI } & \text { Zeta potential (mV) } & \text { EE (\%) }\end{array}$

Time $=0$

$\begin{array}{lcccc}\text { MDPP } & 207 \pm 28^{\mathrm{Ab}} & 0.25 \pm 0.03^{\mathrm{Ac}} & -63.4 \pm 6.5^{\mathrm{A}} & 67.2 \pm 3.2 \\ \text { Unloaded MDPP } & 175 \pm 15^{\mathrm{A}} & 0.27 \pm 0.03^{\mathrm{A}} & -58.9 \pm 4.6^{\mathrm{A}} & - \\ \text { MAPP } & 175 \pm 3^{\mathrm{Ac}} & 0.28 \pm 0.02^{\mathrm{Abc}} & -74.8 \pm 4.0^{\mathrm{A}} & 74.1 \pm 2.7 \\ \text { Unloaded MAPP } & 171 \pm 7^{\mathrm{A}} & 0.29 \pm 0.02^{\mathrm{A}} & -65.5 \pm 2.6^{\mathrm{B}} & - \\ \text { LAPP } & 183 \pm 20^{\mathrm{Abc}} & 0.29 \pm 0.02^{\mathrm{Abc}} & -61.2 \pm 3.9^{\mathrm{A}} & 63.9 \pm 2.9 \\ \text { Unloaded LAPP } & 179 \pm 10^{\mathrm{A}} & 0.28 \pm 0.02^{\mathrm{A}} & -57.5 \pm 4.2^{\mathrm{A}} & - \\ \text { PUPP } & 141 \pm 18^{\mathrm{Ad}} & 0.15 \pm 0.05^{\mathrm{Ae}} & -74.3 \pm 4.6^{\mathrm{A}} & 55.3 \pm 1.8 \\ \text { Unloaded PUPP } & 130 \pm 13^{\mathrm{A}} & 0.17 \pm 0.02^{\mathrm{A}} & -72.5 \pm 3.4^{\mathrm{A}} & -\end{array}$

Time $=30$ days

$\begin{array}{lcccc}\text { MDPP } & 346 \pm 29^{\mathrm{a}} & 0.39 \pm 0.07^{\mathrm{a}} & -61.2 \pm 2.8 & 63.8 \pm 2.0 \\ \text { MAPP } & 195 \pm 12^{\mathrm{bc}} & 0.28 \pm 0.03^{\mathrm{bc}} & -70.7 \pm 5.9 & 74.3 \pm 4.3 \\ \text { LAPP } & 183 \pm 18^{\mathrm{bc}} & 0.32 \pm 0.01^{\mathrm{b}} & -59.3 \pm 3.3 & 56.1 \pm 4.1 \\ \text { PUPP } & 136 \pm 7^{\mathrm{d}} & 0.17 \pm 0.02^{\mathrm{d}} & -67.0 \pm 4.9 & 51.1 \pm 1.4\end{array}$

$P$ values

$\begin{array}{lllll}\text { Sample (loaded powder) } & <0.0001 & <0.0001 & <0.0001 & <0.0001 \\ \text { Time } & <0.0001 & 0.0009 & 0.0266 & 0.0060 \\ \text { Interaction } & <0.0001 & 0.0027 & 0.6357 & 0.1753\end{array}$

Data are means $\pm \mathrm{SD}(\mathrm{n}=3)$ 
${ }^{1}$ MDPP: MD based proniosome powder; MAPP: MA based proniosome powder, LAPP: LA based proniosome powder; PUPP: PU based proniosome powder; PDI: polydispersity index; EE: entrapment efficiency

${ }^{2}$ In each column, means with different small letters show a significant interaction at $\mathrm{P}<0.05$ (Duncan's multiple range test). Different capital letters show significant differences between unloaded and CTX loaded pair for the same carrier after preparation at $\mathrm{P}<0.05$ (Students' t-test) 
Table 2 Micromeritic properties of proniosome powders produced with different carriers ${ }^{1,2}$

\begin{tabular}{lccc}
\hline Sample name & $\theta\left(^{\mathrm{o}}\right)$ & CI $(\%)$ & HR \\
\hline MDPP & $25 \pm 3^{\mathrm{b}}$ & $21 \pm 4^{\mathrm{c}}$ & $1.27 \pm 0.07^{\mathrm{c}}$ \\
MAPP & $33 \pm 2^{\mathrm{a}}$ & $23 \pm 4^{\mathrm{bc}}$ & $1.30 \pm 0.07^{\mathrm{bc}}$ \\
LAPP & $34 \pm 2^{\mathrm{a}}$ & $35 \pm 2^{\mathrm{a}}$ & $1.53 \pm 0.05^{\mathrm{a}}$ \\
PUPP & $31 \pm 2^{\mathrm{a}}$ & $28 \pm 4^{\mathrm{b}}$ & $1.40 \pm 0.09^{\mathrm{b}}$ \\
\hline
\end{tabular}

Data are means \pm SD $(n=3)$

${ }^{1} \theta$ : angle of repose; CI: Carr's compressibility index; HR: Hausner's ratio. For other abbreviations see Table 1

${ }^{2}$ In each column, means with different letters show significant differences at $\mathrm{P}<0.05$ 


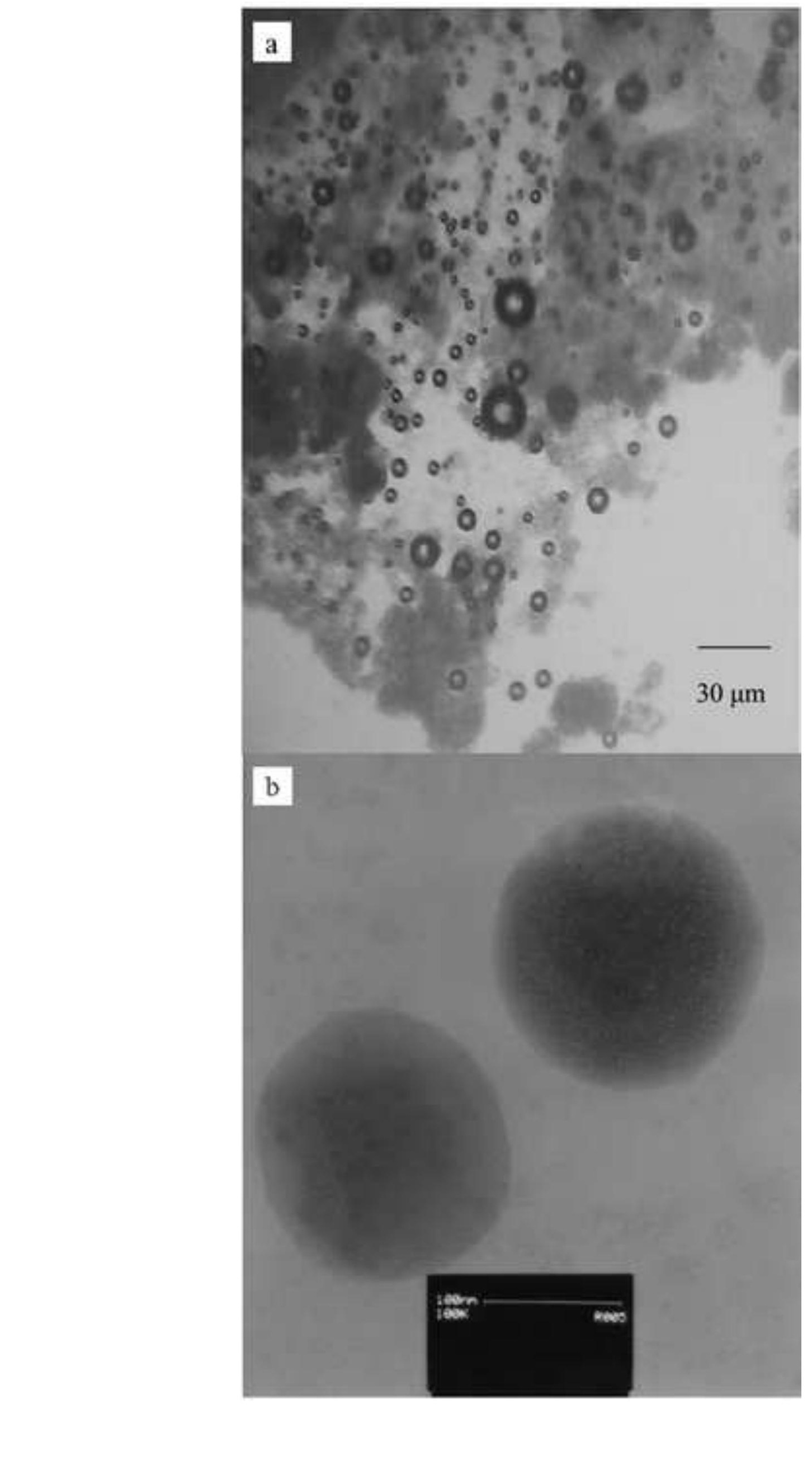

Figure 1
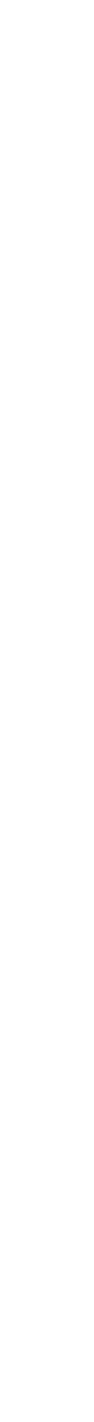

$$
\text { tase }
$$
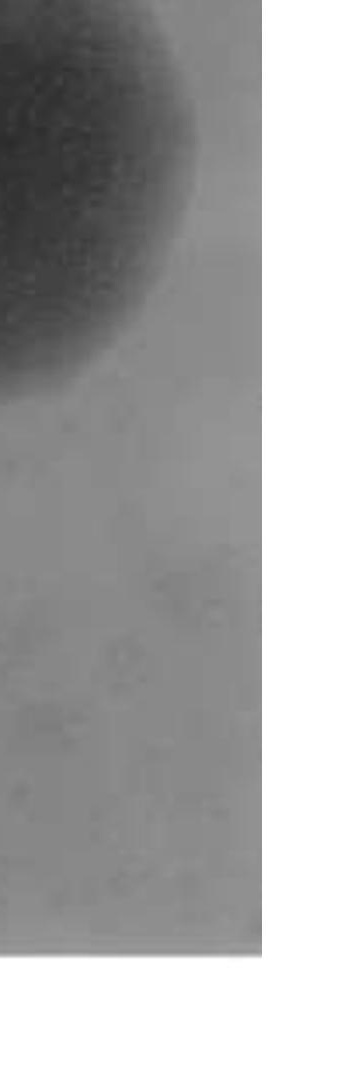


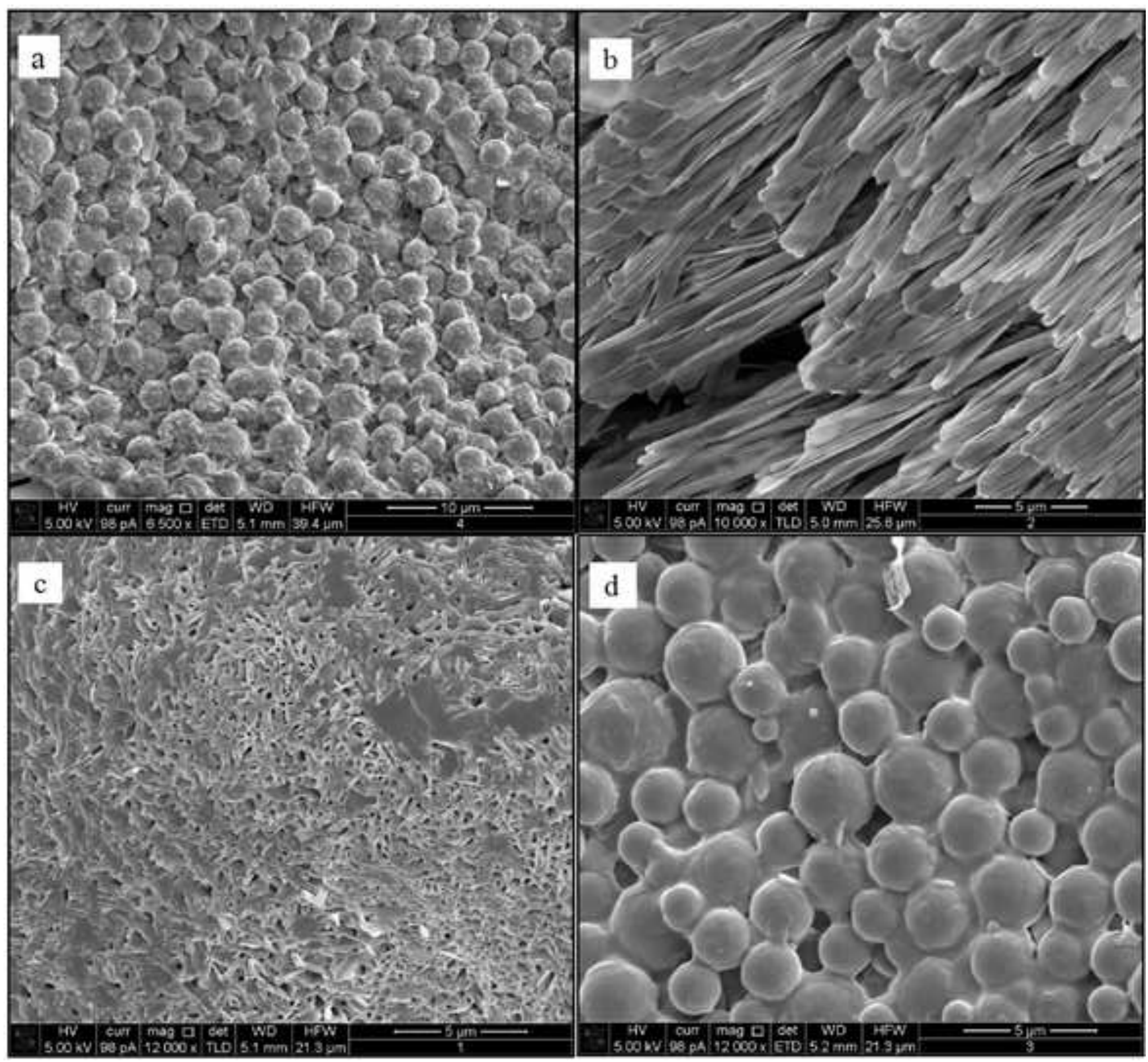

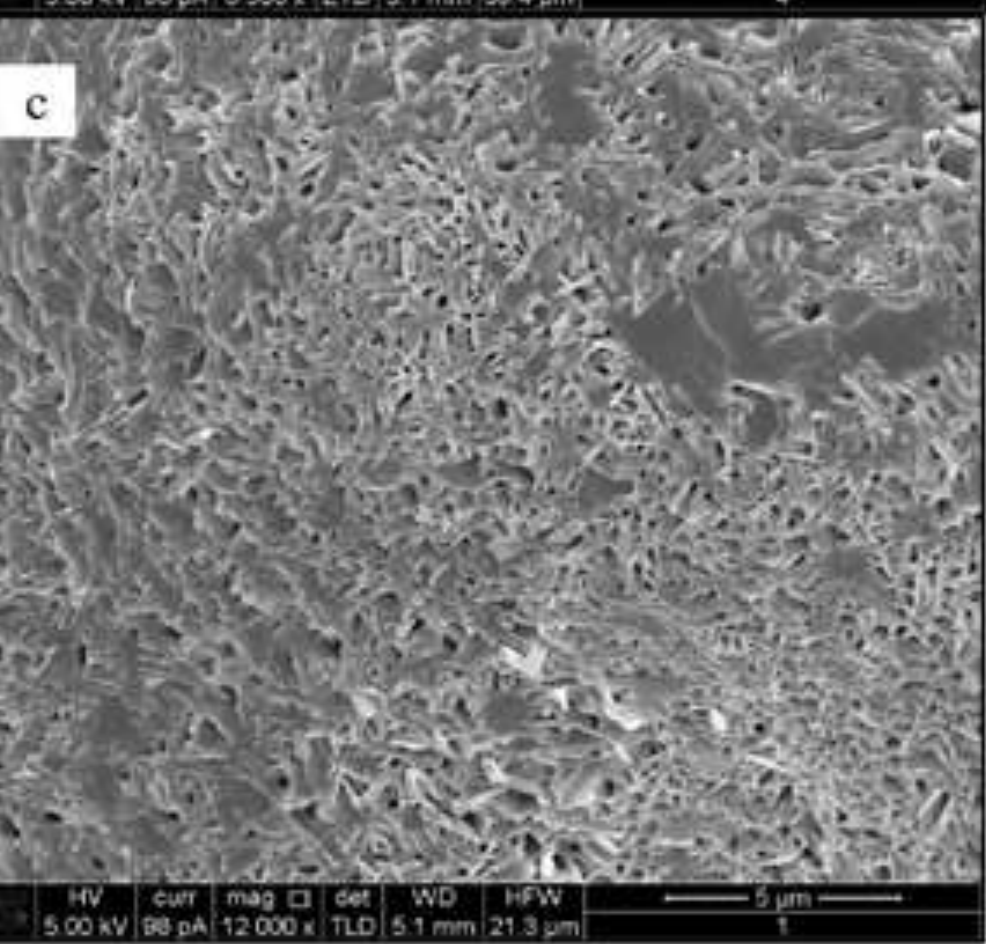

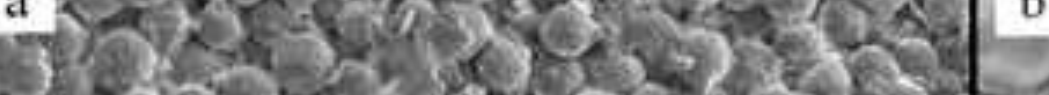

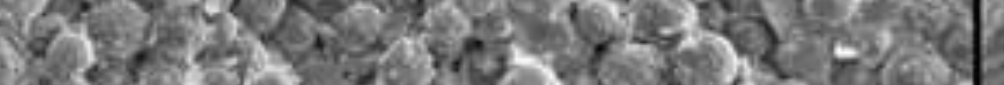

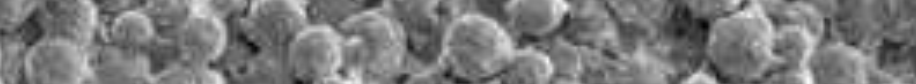

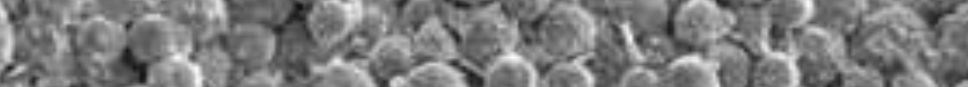

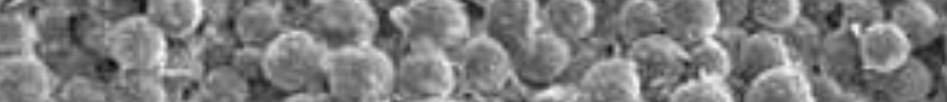
S3.

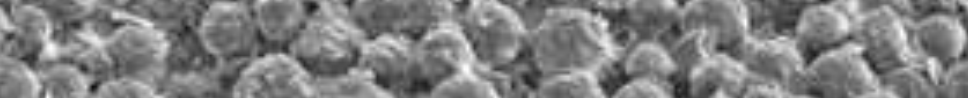

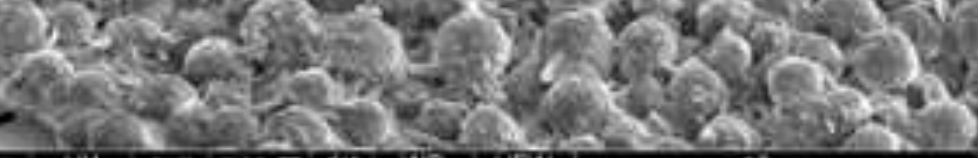




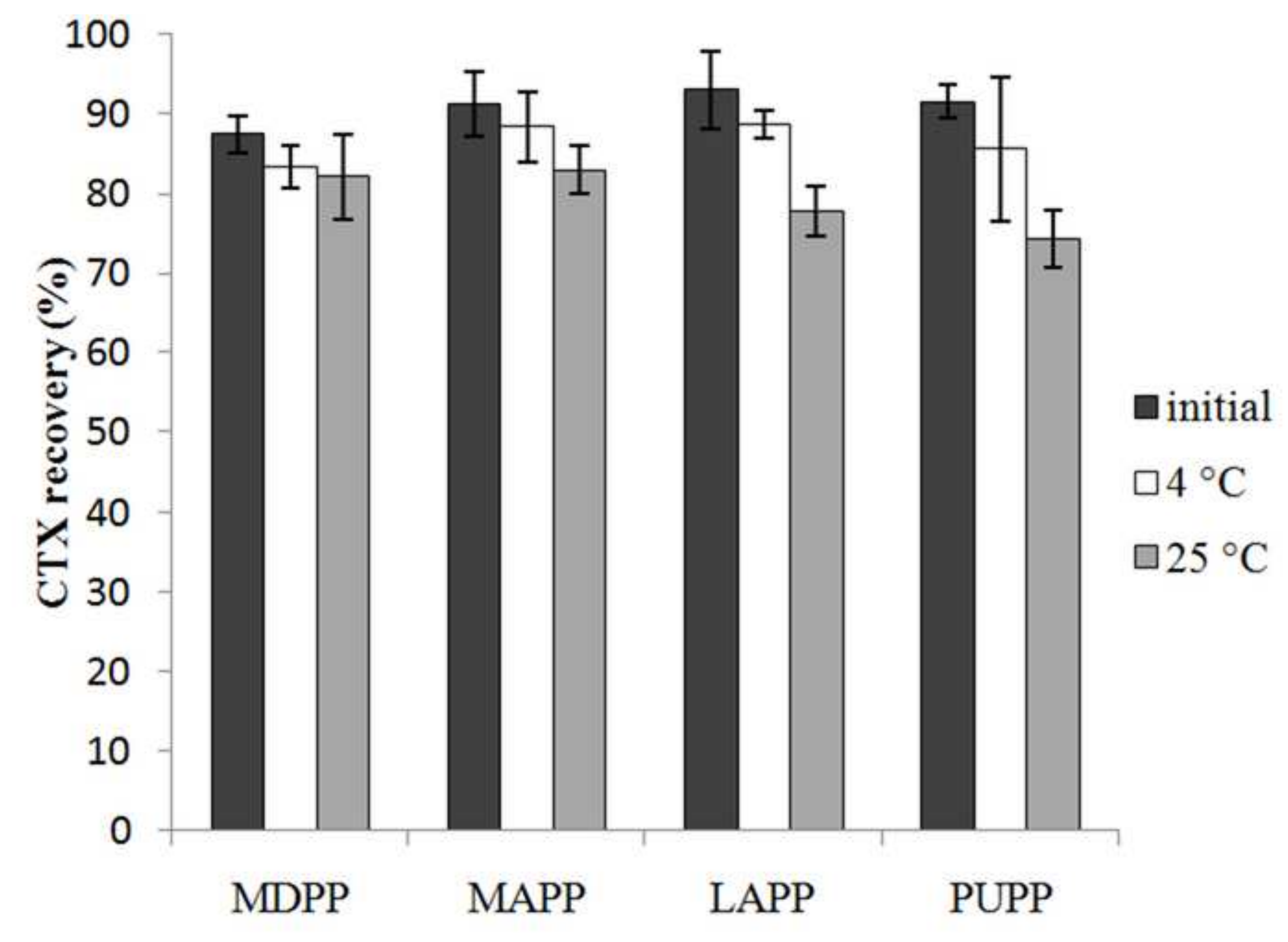




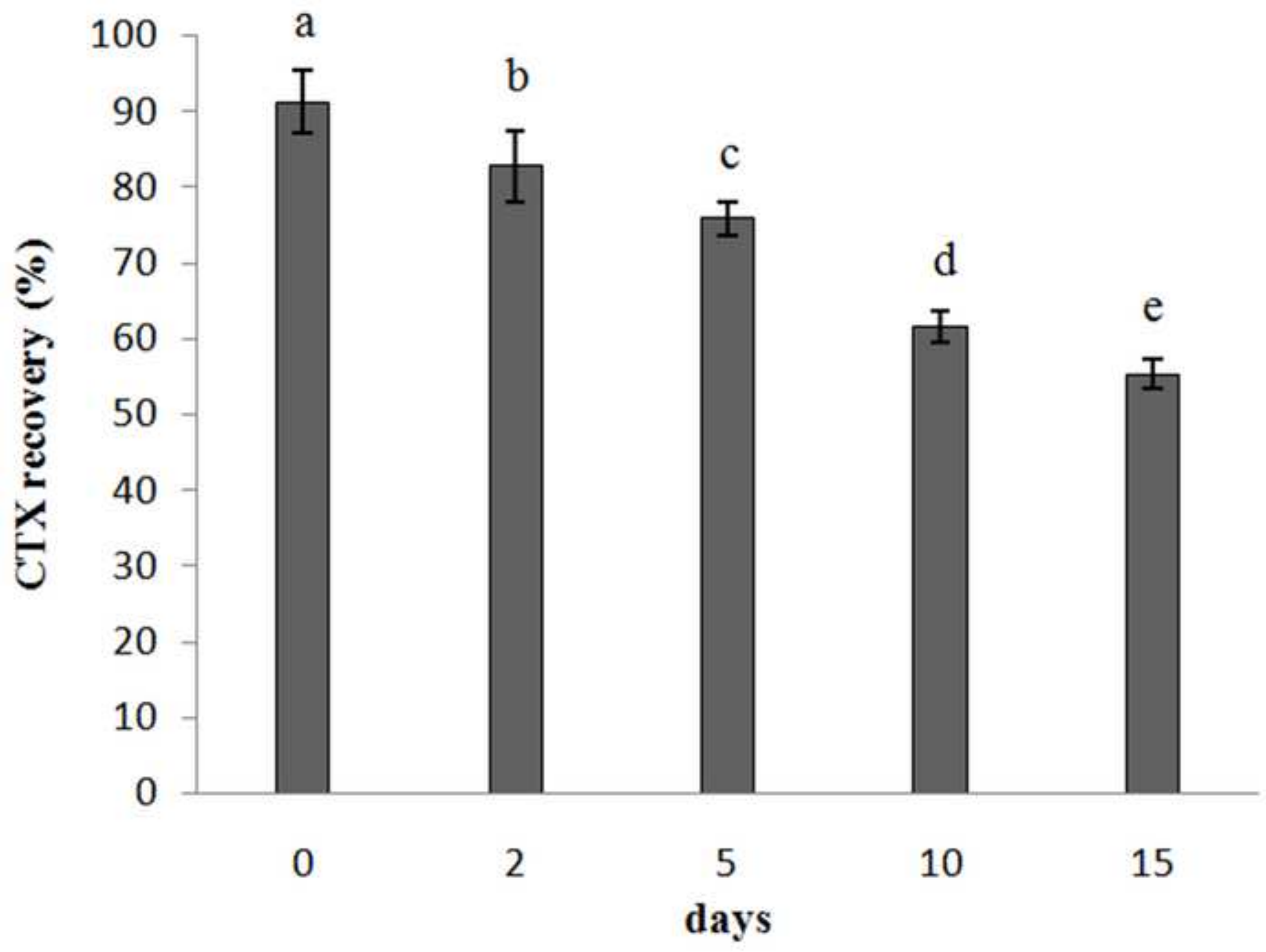

